\section{Accumulation of disadvantage over the life course and mortality}

\author{
Johannes Siegrist
}

Social inequalities in morbidity and mortality continue to be one of the highest public health concerns in times of increasing income inequalities in economically advanced countries. Compelling scientific evidence supports the notion that adverse socioeconomic circumstances determine poor health. To a large extent, respective epidemiological research is based on established indicators of people's socioeconomic position, such as education, occupational status and income. With a few exceptions, wealth, a complementary indicator, has received less attention. The paper by Demakakos et al, ${ }^{1}$ published in the April issue, addresses this gap of knowledge, and it offers several significant insights. First, it demonstrates that accumulated disadvantage over the life course in terms of household wealth (assets, housing) is a powerful predictor of mortality, specifically so in early old age. This finding is derived from a national sample of older men and women, the English Longitudinal Study of Ageing. It is particularly robust as it is based on a large number of incident deaths observed over a 9.4-year follow-up period, as it contains a comprehensive assessment of household wealth measured by 22 indicators at baseline and during follow-up, and as it takes a number of important confounders and potential mediators into account. Second, multivariate analyses reveal that associations of wealth with mortality vary according to cause of death. Whereas strongest associations are observed in case of cardiovascular mortality, no significant relations turn out with cancer-related deaths, where health-related behaviours seem to play a more important role. This observation is in line with a substantial body of knowledge on a far-reaching impact of adverse socioeconomic living and working conditions on the development of cardiovascular diseases.

Correspondence to Professor Johannes Siegrist, Senior Professorship on Work Stress Research, University of Duesseldorf, Duesseldorf, 40225 Germany; johannes.siegrist@med.uni-duesseldorf.de
For scientific as well as policy reasons, a third insight of the Demakakos et al's ${ }^{1}$ paper is of special interest. When studying social determinants of mortality at older age, the conventional socioeconomic position indicators (education, income, occupational status) remain no longer significant predictors of differential mortality once total household wealth is introduced in the model. Scientifically, this offers a challenge to uncover the hidden pathways linking wealth with risks of premature death. In terms of policy, the finding emphasises the adverse long-term effects of accumulated socioeconomic disadvantage from childhood to adulthood, thus supporting programmes and interventions that reduce inequalities at early stages of educational and occupational careers. ${ }^{2}$

To uncover pathways linking household wealth with mortality a closer look at the sample of this study may be instructive. We observe that more than half of older people in the lowest wealth tertile are not married (compared to $19.3 \%$ of the wealthiest tertile), that almost $70 \%$ are members of a low occupational class defined by semiroutine or routine occupations (compared to 21.7\%), and that more than half belong to the tertile with lowest income or pension (compared to 14.4\%). ${ }^{1}$ The fact that each one of these circumstances is associated with elevated mortality risks ${ }^{3-5}$ points to an intriguing web of causation defined by a combination of distinct material and psychosocial adversities. Along these lines an observation from a longitudinal analysis of income and mortality among Canadian men deserves attention: men with a steadily increasing income in their 10 to 20 years of earning histories who nevertheless remained captured in the lowest income group had a higher mortality risk than men with somewhat higher income, whether or not associated with a comparable increase over time. ${ }^{5}$ It may well be that disadvantaged lifetime household wealth reflects people's extensive trajectories of recurrent striving without real success, of being locked in a thoroughly unrewarding social environment. ${ }^{6}$ Such environments are likely to advance states of 'allostatic load' and related morbidity and mortality risks. ${ }^{7}$ Clearly, more research along these lines is needed, but the authors of this new report are to be congratulated for their important and intellectually stimulating contribution.

Competing interests None declared.

Provenance and peer review Commissioned; internally peer reviewed.
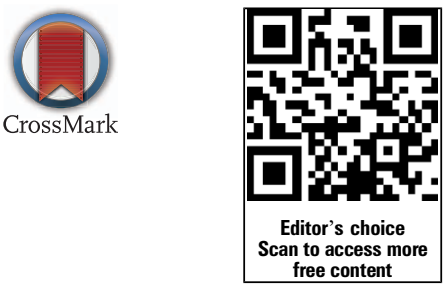

To cite Siegrist J. J Epidemiol Community Health 2016;70:423.

Received 29 October 2015

Accepted 5 November 2015

Published Online First 24 November 2015

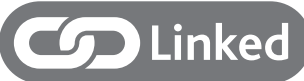

http://dx.doi.org/10.1136/jech-2015-206173

J Epidemiol Community Health 2016;70:423. doi:10.1136/jech-2015-206834

\section{REFERENCES}

1 Demakakos P, Biddulph JP, Bobak M, et al. Wealth and mortality at older ages: a prospective cohort study. J Epidemiol Community Health 2016;70: 346-53.

2 Marmot M, Allen J, Goldblatt P, et al. Fair society, healthy lives. The Marmot Review. Strategic review of health inequalities in England post-2010. http://bit.ly/ S9lHOI

3 Holt-Lunstad J, Smith T, Layton J. Social relationships and mortality risk: a meta-analytic review. PLoS Med 2010;7:e1000316.

4 Marmot MG, Shipley MJ. Do socioeconomic differences in mortality persist after retirement? 25 Year follow up of civil servants from the first Whiitehall study. BMJ 1996;313:1177-80.

5 Wolfson M, Rowe G, Gentleman JF, et al. Career earnings and death: a longitudinal analysis of older Canadian men. J Gerontol Soc Sci 1993;48: S167-79.

6 Siegrist J. Place, social exchange and health: proposed sociological framework. Soc Sci Med 2000;51:1283-93.

7 McEwen BM. Physiology and neurobiology of stress and adaptation: central role of the brain. Physiol Rev 2007:87:873-904. 